\title{
Condição bucal de trabalhadores de fábricas de baterias e uso de serviços odontológicos
}

\author{
Oral health status and use of dental services in battery factory workers
}

\author{
Fábio Duarte da Costa Aznar', Bruno Calzavara', Adriana Rodrigues de Freitas', \\ Fabiano Duarte da Costa Aznar', Sílvia Helena de Carvalho Sales-Peres ${ }^{1}$, Arsenio Sales-Peres ${ }^{1}$
}

\begin{abstract}
RESUMO | Contexto: A Odontologia do Trabalho é responsável por estudar, interpretar e solucionar os diferentes problemas bucais dos trabalhadores, contribuindo para sua qualidade de vida e maior produtividade. Objetivos: Avaliar as condições bucais de trabalhadores em duas fábricas de baterias e o uso de serviços odontológicos em Bauru, São Paulo. Métodos: A amostra foi constituída por 134 indivíduos que responderam a um questionário sobre o uso de serviços. Os exames foram realizados utilizando-se o Índice CPOD para a cárie dentária, Índice Periodontal Comunitário (IPC) e Perda de Inserção Periodontal (PIP) para a doença periodontal, e o Índice de Desgaste Dentário (IDD) (Kappa>0,94). A análise foi descritiva, por meio de frequências absolutas e relativas. Resultados: Com relação ao uso de serviços, 55,22\% relataram consulta odontológica há menos de 1 ano, 76,87\% buscaram atendimento particular/convênio e 33,58\% como consulta de rotina. Apenas 12,60\% dos trabalhadores apresentaram-se livres de cárie, sendo observado CPOD médio de 8,49. Houve presença de cálculo em $56,70 \%$, bolsas rasas em $17,90 \%$ e bolsas profundas em $1,50 \%$ dos trabalhadores. A perda de inserção periodontal foi observada em 26,52\%. Todos os trabalhadores apresentaram desgaste dentário, sendo esse mais prevalente em dentina. Conclusão: Os serviços odontológicos oferecidos pelas empresas pesquisadas propiciaram uma condição bucal satisfatória aos trabalhadores.
\end{abstract}

Palavras-chave I odontologia do trabalho; saúde do trabalhador; serviços de saúde do trabalhador.

\begin{abstract}
Context:Occupational Dentistry is responsible for studying, interpreting and solving the various oral problems of workers, contributing to their quality of life and greater productivity. Objectives: To evaluate the oral conditions of workers in two battery factories and the use of dental services in Bauru, São Paulo, Brazil. Methods: The sample consisted of 134 individuals who responded to a questionnaire on the use of services. The tests were conducted using the DMFT index for dental caries, Community Periodontal Index (CPI) and Periodontal Attachment Loss (PAL) for periodontal disease, and the Dental Wear Index (DWI) (Kappa >0.94). A descriptive analysis was done by means of absolute and relative frequencies. Results: Regarding the use of services, $55.22 \%$ reported dental visits in less than 1 year, $76.87 \%$ sought private care, and $33.58 \%$ as part ofa routine consultation. Only $12.60 \%$ of the workers were free of caries, with mean DMFT of 8.49 . There was calculus in $56.70 \%$, shallow pockets in $17.90 \%$ and deep pockets in $1.50 \%$ of the workers. The periodontal attachment loss was observed in $26.52 \%$. All workers had tooth wear, which was more prevalent in dentin. Conclusion: The dental services offered by the surveyed companies provided a satisfactory oral health to the workers. Keywords | occupational dentistry; occupational health; occupational health services.
\end{abstract}

Trabalho realizado no Departamento de Odontopediatria, Ortodontia e Saúde Coletiva, Faculdade de Odontologia de Bauru, Universidade de São Paulo (USP) Bauru (SP), Brasil.

'Departamento de Odontopediatria, Ortodontia e Saúde Coletiva, Faculdade de Odontologia de Bauru, USP - Bauru (SP), Brasil.

DOI: $10.5327 / Z 1679-443520163415$ 


\section{INTRODUÇÃO}

A saúde e a segurança dos trabalhadores são de vital importância para o bom funcionamento de todos os setores de uma empresa. Colaboradores despreparados, distraídos e não focados em suas funções podem comprometer o processo de produção gerando, como consequência, a queda da produtividade. As condições do meio ou do ambiente de trabalho estão intimamente relacionadas com o processo saúde-doença dessa população, influenciando no desenvolvimento de alterações e problemas bucais, como pigmentação de dentes e mucosa, infecções, traumas e processos patológicos ${ }^{1}$.

O campo da saúde bucal do trabalhador, cujos princípios se aproximam aos da saúde do trabalhador, tem como objeto a relação entre saúde bucal e trabalho, tratando de promover, preservar e recuperar a saúde bucal de populações inseridas nos diversos processos de trabalho contribuindo, assim, para a sua qualidade de vida ${ }^{2}$. Desse modo, haja vista os distúrbios à saúde do trabalhador decorrentes do ambiente laboral, a Odontologia do Trabalho é a especialidade odontológica imprescindível para a harmonia do ambiente de trabalho, seja pela preservação da saúde do trabalhador, seja pela manutenção desses em suas funções, garantindo a produtividade da empresa. Para isso, deve-se ter conhecimento sobre os problemas bucais que podem acometer os trabalhadores, fazendo análises epidemiológicas e etiológicas, possibilitando a compreensão dos impactos bucais em sua qualidade de vida ${ }^{3,4}$.

A boca é a porta de entrada do sistema digestório, atuando também de forma auxiliar na respiração. Em virtude de sua localização e das funções que exerce, é uma zona de absorção, retenção e excreção de substâncias tóxicas que penetram no corpo, estando sujeita a agressões de diferentes naturezas ${ }^{5}$. Sabe-se que, no ambiente do trabalho, o homem está exposto a um conjunto diversificado de agentes que podem causar danos à saúde. Nesse sentido, as condições de trabalho são importantes para a preservação das estruturas bucais, podendo desencadear uma série de doenças em consequência de exposições de natureza ocupacional ${ }^{6}$.

Trabalhadores de fábricas de bateria estão expostos a névoas ácidas, decorrentes do manuseio de materiais potencialmente tóxicos, dentre eles, o chumbo. Dados obtidos em estudos evidenciaram a associação que pode existir entre exposição ocupacional e a ocorrência de problemas bucais em trabalhadores desse setor da indústria ${ }^{7-9}$.

Sendo assim, a finalidade do presente estudo foi avaliar as condições bucais de trabalhadores em duas fábricas de baterias e o uso de serviços odontológicos no município de Bauru, São Paulo.

\section{MÉTODOS}

Um estudo transversal descritivo foi conduzido para a avaliação das condições de saúde bucal de trabalhadores do setor metalúrgico - fábricas de baterias automotivas - e do uso de serviços odontológicos do município de Bauru, São Paulo. O protocolo de pesquisa do estudo foi aprovado pelo Comitê de Ética em Pesquisa da Faculdade de Odontologia de Bauru da Universidade de São Paulo (USP) (Processo no 48495/2012).

O cálculo amostral deste estudo foi realizado utilizando-se a diferença entre duas proporções, com efeito de desenho de $5 \%$ e erro de $20 \%$. Foram utilizados os dados do Levantamento Nacional de Saúde Bucal SB Brasil 2010 relativos à prevalência de bolsas periodontais (rasas e profundas - 19,4\%) na população adulta ( 35 a 44 anos) ${ }^{10}$ e dados sobre a prevalência de contaminação por chumbo $(43,4 \%)$ em trabalhadores de fábricas e de reciclagem de baterias automotivas ${ }^{11}$. Utilizando-se esses parâmetros, segundo o efeito de desenho, obteve-se amostra de 134 indivíduos - trabalhadores de duas fábricas de bateria na cidade de Bauru, São Paulo.

Após os esclarecimentos sobre os métodos, riscos e benefícios da participação na pesquisa, os trabalhadores de ambos os gêneros, com idades entre 16 e 56 anos, foram incluídos na amostra após a assinatura do Termo de Consentimento Livre e Esclarecido. Os critérios de exclusão adotados foram o edentulismo total e o uso de medicamentos anti-inflamatórios, antibióticos e anticonvulsivantes.

O estudo foi composto por duas etapas: aplicação de questionário e exame clínico, realizados em sequência. O questionário aplicado - baseado no utilizado pelo SB Brasil $2010^{10}$ - foi composto por questões sobre o uso de serviços odontológicos, sendo de fácil entendimento pelos entrevistados, uma vez que as perguntas formuladas foram respondidas e devolvidas sem haver a interferência dos pesquisadores. Os questionamentos, em um total de quatro, consistiram nas seguintes perguntas: 
- Alguma vez na vida o(a) $\operatorname{Sr}(a)$. já foi ao consultório de um cirurgião-dentista?

- Quando consultou o cirurgião-dentista pela última vez?

- Onde foi a última consulta?

- Qual o motivo da sua última consulta?

Os exames clínicos foram conduzidos por dois examinadores devidamente treinados e calibrados. Foi calculado o coeficiente Kappa intraexaminador - com o re-teste em $10 \%$ da amostra ${ }^{12}$-, cujo resultado foi de 0,94. A coleta de dados foi realizada nas dependências das empresas participantes, sob iluminação natural, com auxílio de espelhos bucais planos no 5 e sondas periodontais tipo point ball ${ }^{12}$.

Os índices utilizados foram:

- Índice CPOD para a cárie dentária;

- Índice Periodontal Comunitário (IPC) e Perda de Inserção Periodontal (PIP) para a doença periodontal ${ }^{13}$; e

- ÍndicedeDesgasteDentário(IDD) parao desgaste dentário ${ }^{14}$.

A análise dos dados foi realizada de forma descritiva, por meio de frequências absolutas e relativas.

\section{RESULTADOS}

A amostra dos trabalhadores avaliados foi constituída por $88,06 \%$ de indivíduos do sexo masculino e $11,94 \%$ do sexo feminino, que apresentavam uma faixa etária média

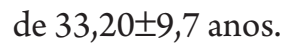

Na aplicação do questionário - composto por questões sobre o uso de serviços odontológicos - observou-se que a grande maioria dos trabalhadores (90,30\%) já havia frequentado um cirurgião-dentista ao menos uma vez na vida, sendo que $55,22 \%$ tinham sido consultados há menos de 1 ano. Os mesmos relataram que a última consulta foi realizada por meio de um plano de saúde ou convênio $(40,30 \%)$, serviço particular $(36,57 \%)$ ou serviço público (14,93\%). Com relação ao motivo dessa última consulta, 33,58\% disseram que a finalidade foi realizar uma revisão (check-up) e $22,85 \%$ para realizar tratamento.

Em relação aos dados obtidos no exame bucal, a média encontrada para o CPOD foi de 8,49 $\pm 5,71$. A decomposição do CPOD encontrado mostra que a média de dentes cariados foi de $0,52 \pm 0,85$, a de dentes perdidos de $1,75 \pm 2,53$ e a de dentes obturados examinados de 6,22 $\pm 5,03$, sendo o último o dado mais relevante na composição do CPOD (Gráfico 1).

Dos participantes, $12,6 \%$ se mostraram livres de cárie, $29,1 \%$ apresentaram pelo menos 1 dente cariado, 52,2\% apresentaram ao menos 1 dente perdido por cárie e 80,6\% tinham pelo menos 1 dente restaurado na boca (Gráfico 2).

Segundo o IPC, o número de sujeitos que apresentaram índice 0 (zero) - que representa nenhum problema de origem periodontal - correspondeu a $22,4 \%$ da amostra. A porcentagem de colaboradores que exibiu índice 3 correspondente à bolsa periodontal de 4 a $5 \mathrm{~mm}$ - foi de $17,9 \%$ e a porcentagem desses que exibiu índice $4-$ bolsa periodontal de $6 \mathrm{~mm}$ ou mais — foi de 1,5\%. Nenhuma pessoa apresentou apenas sangramento gengival após sondagem,

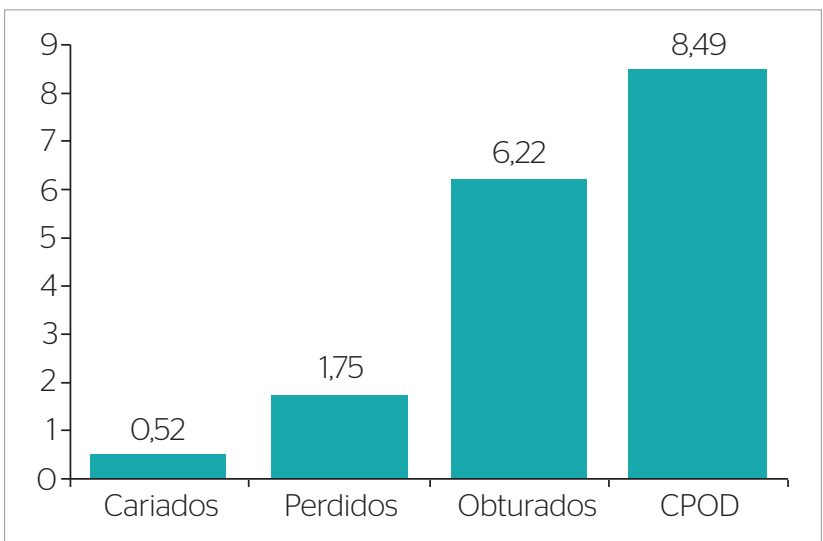

Gráfico 1. Média de dentes cariados, perdidos, obturados e escore geral do índice de cárie dentária.

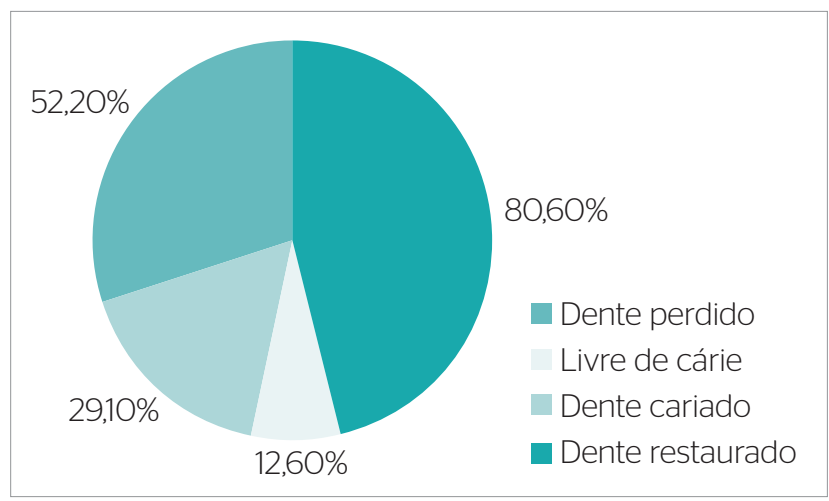

Gráfico 2. Distribuição das condições da coroa em relação à cárie dentária. 
referente ao índice 1 do IPC. Entretanto, 25\% dos sujeitos avaliados mostraram sangramento após sondagem associado a outro problema periodontal de maior gravidade. O índice 2 do IPC — que trata apenas da presença do cálculo - , foi detectado em $56,7 \%$ dos colaboradores. Todavia, a porcentagem total de cálculo encontrada, seja ela associada a outro problema ou não, foi de $75 \%$ (Gráfico 3 ).

O índice PIP, que avalia a perda de inserção periodontal tendo como base a junção cemento-esmalte, mostra que 73,48\% da amostra observada apresentou índice 0 (zero), ou seja, perda de inserção de 0 a $3 \mathrm{~mm}$, que configura uma situação de normalidade; $22,73 \%$ apresentaram uma perda de inserção em concordância com o índice 1 - perda de inserção de 4 a $5 \mathrm{~mm}$. As perdas de inserção mais severas, caracterizadas pelos índices 2 - perda de inserção de 6 a $8 \mathrm{~mm}$ - 3 perda de inserção de 9 a $11 \mathrm{~mm}$ - e 4 - perda de inserção de $12 \mathrm{~mm}$ ou mais - representam apenas 3,79\% do total dos trabalhadores avaliados respectivamente (Gráfico 4).

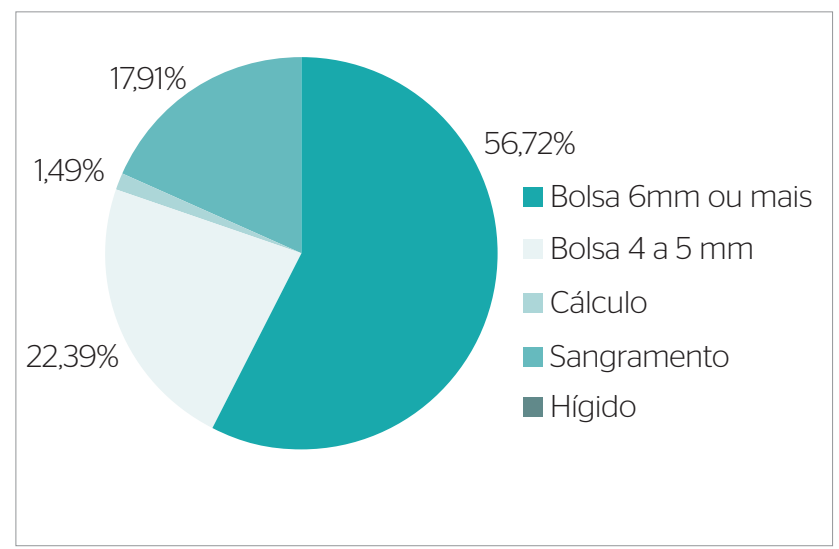

Gráfico 3. Índice Periodontal Comunitário.

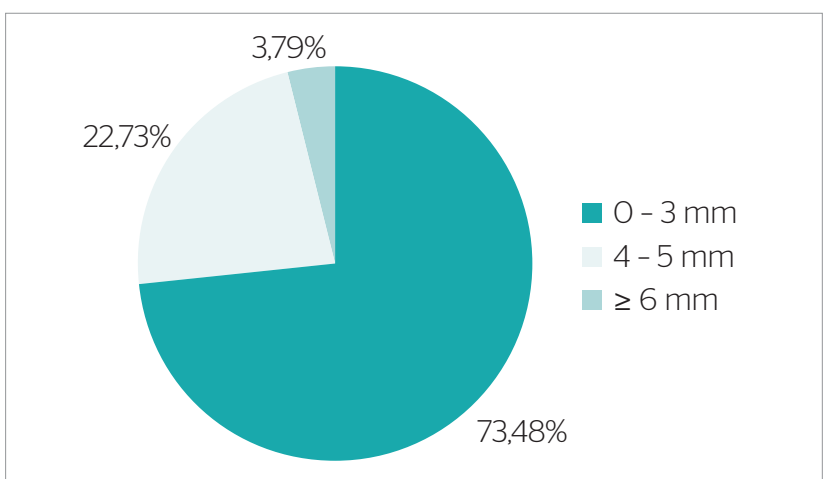

Gráfico 4. Perda de Inserção Periodontal.
Quando ao IDD, foi detectado desgaste das estruturas minerais dentárias em 100\% dos indivíduos observados. Cerca de $19 \%$ da amostra apresentou dentes com desgaste tipo 1 - desgaste incipiente, somente em esmalte -, 70\% mostrou dentes com desgaste tipo 2 - desgaste moderado, envolvendo dentina - e $11 \%$ dos trabalhadores exibiram dentes com desgaste tipo 3 - desgaste severo, se estendendo até a polpa - (Gráfico 5). Dentre os dentes avaliados, os mais acometidos pelo desgaste em dentina foram os incisivos (48\%), seguidos por caninos (33\%), prémolares (14\%) e molares (5\%). Com relação ao desgaste em esmalte, apresentaram maior acometimento os incisivos $(31 \%)$, seguidos pelos molares $(30 \%)$, pré-molares (27\%), e caninos (11\%) (Gráfico 5).

\section{DISCUSSÃO}

Os dados obtidos revelaram que a amostra estudada foi composta predominantemente por trabalhadores do sexo masculino, provavelmente devido ao tipo do trabalho realizado na fabricação das baterias automobilísticas. A média de idade encontrada foi de 33,20 anos - em sua maioria na faixa etária entre 20 e 40 anos - , o que condiz com $78 \%$ dos participantes da pesquisa. Em 2011, o Ministério do Trabalho e Emprego, citando levantamento realizado em 2009 pela Relação Anual de Informações Sociais (RAIS) ${ }^{15}$, relatou que a maior parcela de trabalhadores ativos no Brasil esteve situada na faixa entre 30 e 39 anos, demonstrando que a amostra estudada neste trabalho pertence a um grupo de pessoas que se encontra em plena atividade laborativa e,

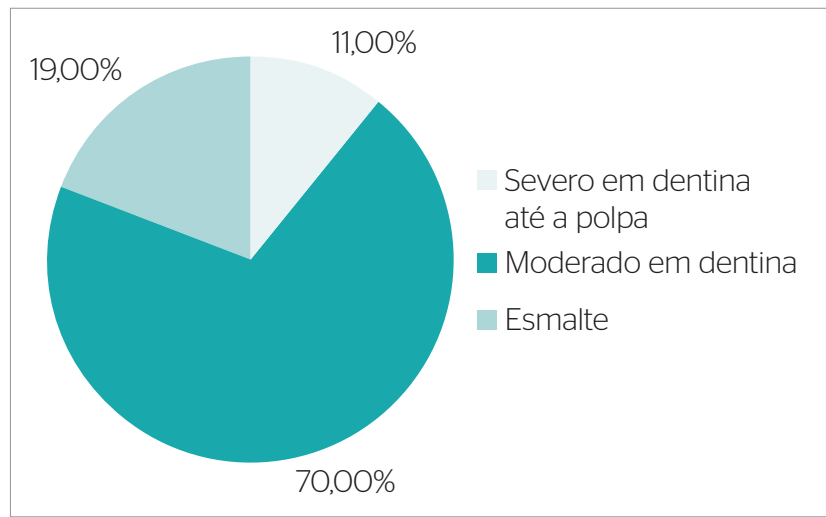

Gráfico 5. Severidade do desgaste dentário observado em $100 \%$ dos trabalhadores. 
portanto, sujeita aos acidentes e às doenças causadas pelas condições de trabalho.

A utilização de serviços está associada a diversos fatores, tais como o nível econômico, educacional e social, bem como padrões de cultura e tradição popular ${ }^{16}$. Nessa pesquisa, no questionário aplicado sobre o uso de serviços odontológicos, $76,86 \%$ dos trabalhadores afirmaram terem consultado um cirurgião-dentista há menos de 2 anos. Apenas 10\% dos sujeitos relataram que a última consulta ao dentista foi por motivo de dor, $34 \%$ passaram por consulta de rotina (check-up) e $30 \%$ por tratamento que não fosse extração. Isso pode sugerir que o tipo de tratamento que os funcionários buscam está relacionado à estética. Embora as diferenças nas necessidades em saúde não sejam eliminadas apenas com o uso de serviços de saúde, é inegável que o acesso a serviços de qualidade pode amenizar condições desfavoráveis de saúde nas populações.

Em relação à cárie dentária, a média do CPOD encontrada nos trabalhadores foi de 8,49; para a faixa etária padrão dos estudos nacionais - 35 a 44 anos - encontramos a média de 10,49. Esse dado demonstra que a condição bucal apresentou-se melhor do que a encontrada nos levantamentos SB Brasil $2003^{17}$ e SB Brasil $2010^{10}$ para a mesma faixa etária, cujas médias foram de 20,13 e 16,75, respectivamente. Outro dado que revela a melhor condição dos trabalhadores estudados nesta pesquisa é o número de indivíduos livres de cárie, $12,6 \%$ da amostra. O projeto SB Brasil 2010 constatou que apenas $0,9 \%$ do total de pessoas entre 35 e 44 anos mostrava-se com CPOD igual a 0 (zero).

Quanto aos componentes do índice CPOD, o que se mostrou mais expressivo na amostra estudada foi a quantidade de dentes restaurados, sendo que $80,6 \%$ dos sujeitos tinham pelo menos 1 dente restaurado na boca, estando esses resultados de acordo com outras pesquisas que avaliaram a mesma faixa etáriaa ${ }^{18,19}$. As melhores condições de saúde bucal em relação à cárie encontradas na amostra do presente estudo podem ser explicadas pelo fato das duas fábricas pesquisadas possuírem programa de saúde bucal, no qual os funcionários recebem tratamento gratuito para procedimentos menos complexos.

Foi observada maior prevalência de cárie dentária e dentes perdidos por cárie em indivíduos com faixa etária mais avançada, quando comparados com aqueles de idade inferior, seguindo o achado de outros pesquisadores ${ }^{20}$. A média mais baixa para $\operatorname{CPOD}(1,67)$ foi apresentada pelos indivíduos com idades entre 16 e 19 anos. Alguns autores afirmam que houve uma queda na prevalência de cárie dentária em vários países nas últimas décadas, apontando que a prevalência da cárie diminui à medida que melhora a condição socioeconômica, mesmo em áreas sem a adição de flúor à agua de abastecimento público ${ }^{21,22}$. Como consequência disso, atualmente há um maior número de jovens e adultos jovens livres de cárie ou com níveis de CPOD baixos ${ }^{23,24}$. Portanto, o que se pode inferir é que as diferenças de CPOD entre as faixas etárias podem ser resultado de mudanças históricas ocorridas na sociedade e também em razão das diferenças entre os níveis socioeconômicos.

As condições do ambiente e de determinados tipos de trabalho podem influenciar a saúde dos tecidos periodontais. Alguns estudos investigaram a exposição a produtos ácidos no ambiente de trabalho como sendo um fator de risco ao desenvolvimento de alterações no periodonto e observaram associação positiva entre essa exposição e problemas ocorridos nos tecidos de sustentação, como sangramento gengival e bolsas periodontais ${ }^{8,25,26}$.

Quanto às condições gengivais, os dados encontrados nos colaboradores das empresas revelam que 22,4\% da amostra esteve livre de qualquer problema periodontal. Observou-se grande prevalência de cálculo dentário, porém, pequena quantidade de sujeitos com bolsas profundas. Esses achados estão em concordância com outros trabalhos nacionais ${ }^{27,28}$. Os resultados encontrados no presente estudo, quando comparados aos encontrados no levantamento SB Brasil 2010 na mesma faixa etária, mostram que a condição periodontal desses trabalhadores é melhor do que a da média nacional da população que se encontra em atividade laborativa para todas as categorias desse índice ${ }^{10}$. Porém, quando comparamos os resultados com o levantamento SB Brasil 2003, observou-se que a condição periodontal piorou de 2003 para 2010. Esse fato pode ter relação com a forma do envelhecimento da população brasileira ${ }^{17,28,29}$.

Para o desgaste dentário, $100 \%$ da amostra apresentou algum tipo de desgaste nos dentes. Contudo, estava presente de forma leve e moderada em $89 \%$, sugerindo que sua causa seja fisiológica, pois é um processo natural, que pode ocorrer com o envelhecimento ${ }^{30,31}$. Em contraposição, alguns autores afirmam que a exposição ocupacional a névoas ácidas atinge o tecido dentário e pode causar perda mineral, demonstrando que esses riscos podem estar mais relacionados às indústrias de bateria e de galvanização ${ }^{6,32}$. 
Autores afirmam que a exposição constante aos produtos químicos do ambiente de trabalho está fortemente associada a uma erosão dentária severa, sendo possível observar áreas com presença de dentina secundária e também áreas afetadas localizadas na região da polpa ${ }^{33}$. No presente estudo, apenas $11 \%$ dos trabalhadores apresentaram desgaste severo, representando somente 2,5\% do total de dentes avaliados, sendo o grupo dentário mais acometido o dos incisivos, tanto em esmalte quanto em dentina. Essas evidências sugerem que o desgaste apresentado por esses indivíduos não tem relação com a atividade realizada no trabalho. Estudos afirmam que o estresse ocupacional é um problema que pode estar associado ao surgimento de diversos processos patológicos bucais, sendo um deles o desgaste dentário ${ }^{34}$.

\section{CONCLUSÕES}

Conclui-se que a condição bucal dos trabalhadores avaliados neste estudo demonstrou-se favorável, fato que pode ser explicado devido à assistência odontológica oferecida pelas empresas pesquisadas, na qual os funcionários receberam tratamento para procedimentos menos complexos.

\section{REFERÊNCIAS}

1. Peres AS, Olympio KPK, Cunha LSC, Bardal PAP. Odontologia do Trabalho e Sistema Único de Saúde: uma reflexão. Rev ABENO. 2004:4(1):38-41.

2. Araújo ME, Marcucci G. Estudo da prevalência das manifestações bucais decorrentes de agentes químicos no processo de galvanoplastia: sua importância para a área de saúde bucal do trabalhador. Odontologia e Sociedade. 2000;2(1/2):20-5.

3. Araújo ME, Gonini Junior A. Saúde bucal do trabalhador: os exames admissional e periódico como um sistema de informação em saúde. Odontologia e Sociedade. 1999;1(1/2):15-8.

4. Macedo IAB, Costa SS. Saúde bucal e sua influência na qualidade de vida do trabalhador: uma revisão de artigos publicados a partir do ano de 1990. Rev Bras Med Trab. 2015;13(1):2-12.

5. Aznar-Longares G, Nava R. Riesgos bucodentales de los trabajadores. Práctica Odontológica. 1988;9:10-18.

6. Vianna MIP, Santana VS. Exposição ocupacional a névoas ácidas e alterações bucais: uma revisão. Cad Saúde Pública. 2001;17(6):1335-44.

7. Petersen PE, Gormsen C. Oral conditions among German battery factory workers. Community Dent Oral Epidemiol. 1991;19(2):104-6.

8. Almeida TF, Vianna MIP, Santana VS, Gomes Filho IS. Occupational exposure to acid mists and periodontal attachment loss. Cad Saúde Pública. 2008;24(3):495-502.

9. Spinola AG, Prata PR. Uso de biomarcadores como indicadores do histórico de exposição ocupacional ao chumbo. Rev Bras Med Trab. 2009;4,5,6:39-45.

10. Brasil. Ministério da Saúde. Secretaria de Atenção à Saúde/ Secretaria de Vigilância em Saúde. SBBrasil 2010: Pesquisa Nacional de Saúde Bucal - Resultados Principais. Brasília: Ministério da Saúde, 2011. 92 p.

11. Minozzo R, Wagner SC, Santos CH, Deimling LI, Mello RS. Prevalência de anemia em trabalhadores expostos ocupacionalmente ao chumbo. Rev Bras Hematol Hemoter. 2009;31(2):94-7.

12. Landis JR, Koch GG. Biometrics. The measurement of observer agreement for categorical data. Biometrics. 1977;33(1):159-74.
13. World Health Organization (WHO). Oral health surveys: basic methods. Geneva: WHO; 1997.

14. Sales-Peres SHC, Goya S, Araújo JJ, Sales-Peres A, Lauris JR, Buzalaf MA. Prevalence of dental wear among 12-year-old Brazilian adolescents using a modification of the tooth wear index. Public Health. 2008;122(9):942-8.

15. Brasil. Ministério do Trabalho e Emprego. Manual de Orientação da Relação Anual de Informações Sociais (RAIS): ano-base 2011. Brasil: MTE, SPPE, DES, CGET; 2012. 60 p.

16. Camargo MBJ, Dumith SC, Barros AJD. Uso regular de serviços odontológicos entre adultos: padrões de utilização e tipos de serviços. Cad Saúde Pública. 2009;25(9):1894-906.

17. Brasil. Ministério da Saúde/Secretaria de Atenção à Saúde. Projeto SBBrasil 2003: condições de saúde bucal da população brasileira 2002-2003: resultados principais. Brasília: Ministério da Saúde, 2004. 68 p.

18. Silva DD, Sousa MLR, Wada RS. Saúde bucal em adultos e idosos na cidade de Rio Claro, São Paulo, Brasil. Cad Saúde Pública. 2004:20(2):626-31

19. Batista MJ, Silva DD, Sousa MLR. Saúde bucal em uma população de adultos no município de Paulínia, São Paulo. Rev Odontol UNESP. 2010;39(4):185-91.

20. Frazão P, Antunes JLF, Narvai PC. Perda dentária precoce em adultos de 35 a 44 anos de idade. Estado de São Paulo, Brasil, 1998. Rev Bras Epidemiol. 2003;6(1):49-57.

21. Jones $\mathrm{CM}$, Worthington $\mathrm{H}$. Water fluoridation, poverty and tooth decay in 12-year-old children. J Dent. 2000;28(6):389-93.

22. Marcenes W, Bonecker MJS. Aspectos epidemiológicos e sociais das doenças bucais. In: Lascala NT. Promoção de saúde bucal na clínica odontológica. São Paulo: Artes Médicas; 2000.

23. Cypriano S, Sousa MLR, Rihs LB, Wada RS. Saúde bucal dos pré-escolares, Piracicaba, Brasil, 1999. Rev Saúde Pública. 2003;37(2):247-53.

24. Marthaler TM. Changes in dental caries 1953-2003. Caries Res. 2004;38(3):173-81. 
25. Tuominen ML. Occurrence of periodontal pockets and oral soft tissue lesions in relation to sulfuric acid fumes in the working environment. Acta Odontol Scand. 1991;49(5):261-6.

26. Amin WA, Al-Omoush SA, Hattab FN. Oral health status of workers exposed to acid fumes in phosphate and battery industries in Jordan. Int Dent J. 2001;51(3):169-74.

27. Gesser HC, Peres MA, Marcenes W. Condições gengivais e periodontais associadas a fatores socioeconômicos. Rev Saúde Pública. 2001;35(3):289-93.

28. Abreu MH, Pereira MLS, Marasco AM, Silva MRP, Tavares AQST, Miyazaki LCY, et al. Contaminação por chumbo em Bauru: vigilância sanitária e ações ambientais no período de 2002 a 2007. Saúde e Sociedade. 2009;18(Suppl 1):83-84.

29. Gaião LR, Almeida MEL, Heukelbach J. Perfil epidemiológico da cárie dentária, doença periodontal, uso e necessidade de prótese em idosos residentes em uma instituição na cidade de Fortaleza, Ceará. Rev Bras Epidemiol. 2005;8(3):316-23.
30. Walters PA. Dentinal hypersensitivity: a review. J Contemp Dent Pract. 2005;6(2):107-17.

31. Bartlett D. A new look at erosive tooth wear in elderly people. J Am Dent Assoc. 2007;138:21S-5S.

32. Wiegand A, Attin T. Occupational dental erosion from exposure to acids: a review. Occup Med (Lond). 2007;57(3):169-76.

33. Kim HD, Hong YC, Koh DH, Paik DI. Occupational exposure to acidic chemicals and occupational dental erosion. J Public Health Dent. 2006;66(3):205-8.

34. Macedo IAB, Oliveira CCC, Costa SS, Silva AM. Manifestações orais do estresse ocupacional: revisão de literatura. Full DentSci.2010;1(3):276-81.

Endereço para correspondência: Fábio Duarte da Costa Aznar -

Departamento de Odontopediatria, Ortodontia e Saúde Coletiva, Faculdade de Odontologia de Bauru, Universidade de São Paulo (USP) -

Alameda Octávio Pinheiro Brisolla, 9-75 - CEP: 17012-901 - Bauru (SP), Brasil -

E-mail: fabio@aznar.com.br 